\title{
Jiggers outbreak in Uganda
}

$\mathrm{A}$

n outbreak of tungiasis, a tropical parasitic skin disease commonly known as jiggers, has killed at least 20 Ugandans and taken host in more than 20000 people in just two months.

Jiggers is caused by the female sand flea, Tunga penetrans, which burrows into the skin of its host - usually on the feet, but occasionally on the lips, buttocks and even eyelids - and undergoes hypertrophy within a day or two, eventually expanding to the size of a pea (www.cdc.gov/NCIDOD/EID/vol9 no8/pdfs/03-0041.pdf). The flea's hindquarters remain in contact with the air, providing an avenue for breathing, defecating and expelling eggs, while creating a painful lesion on the host's skin which often serves as an entry point for pathogenic microorganisms. Over the course of about three weeks, the flea will expel as many as 200 eggs into the environment and then die in situ, leaving a black crust covering a lesion containing the dead flea, which is eventually sloughed off from the epidermis. If left untreated, patients can die of secondary infections such as tetanus and gangrene, according to the CDC.

"Jiggers can easily kill young children by sucking their blood and can cause early deaths in grown-ups who have other diseases. Most of those infected, especially the elderly and children, cannot walk," James Kakooza, Uganda's minister of state for primary health care, told a press conference.

There are low levels of awareness about the cause, mode of spread and prevention of jiggers among Ugandans. In fact, myths surrounding the disease are an obstacle to eradication.

"People must change their attitudes toward the disease and stop believing that this is witchcraft," says Simon Wanjala, a medical official based in eastern Uganda, in an interview. "Now they simply wait and die instead of trying to remove the pests from their bodies, so many of those affected end up seeking treatment from traditional heal-

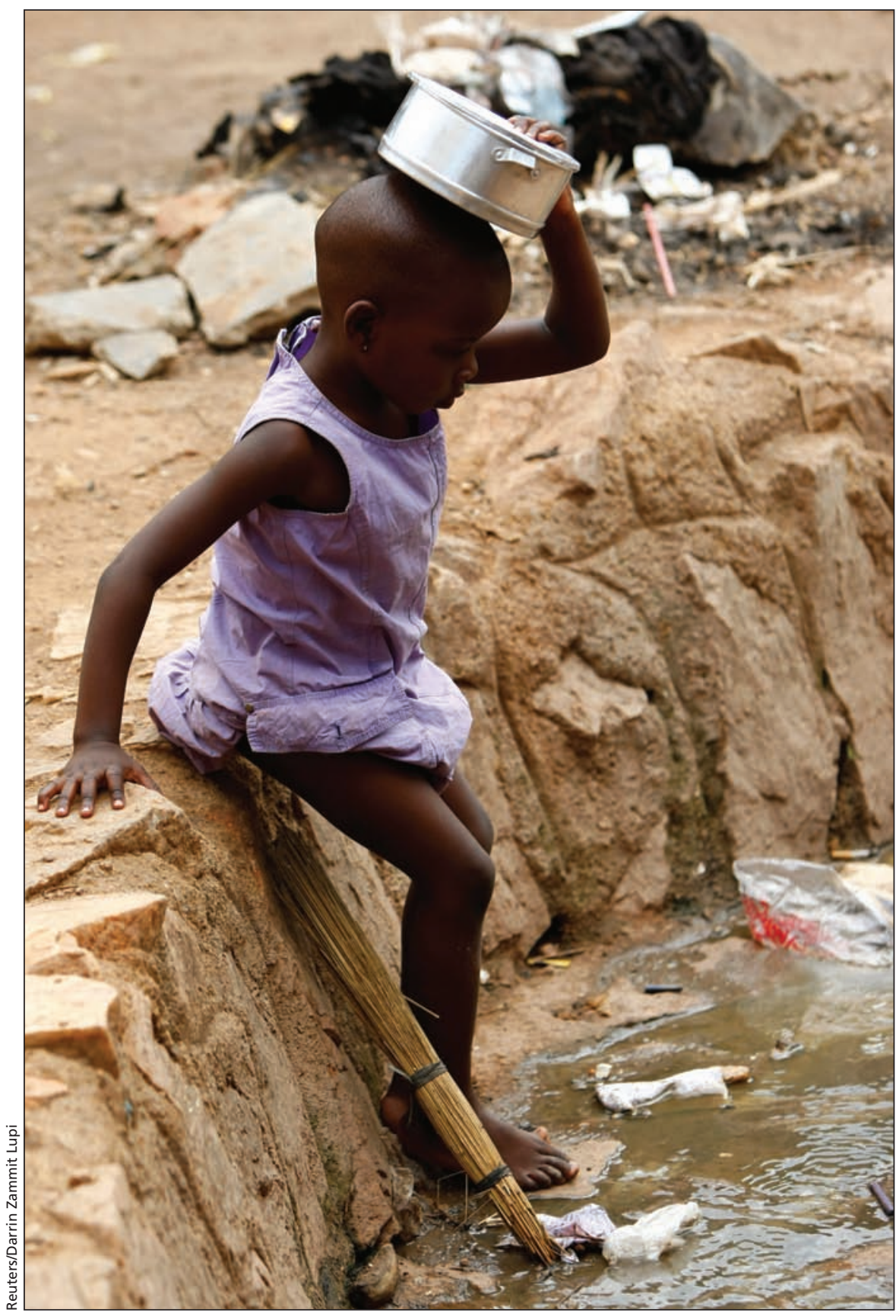

A child steps into an open sewer in Kisenyi, Uganda.

ers instead of seeking conventional health care givers."

Treatment largely involves removal of the flea "with a sterile needle and topical application of an antibiotic in case of secondary infection. If the flea bursts, e.g. during the attempt to extract it with a needle, wire, nail, etc., severe inflammation is inevitable" (Trop Med Int Health 2001;6:267-72). The same study indicates that factors influencing the incidence of the disease include "stray dogs and cats, pigs in close vicinity to living quarters, unpaved streets, 
mud floors in houses, insufficient or non-existent sanitation; and infestation with rats and mice especially in areas without rubbish disposal. Many people, especially children, mostly walk barefoot or only wear slippers. Illiteracy, ignorance and neglect presumably are other factors favouring the high prevalence of severe pathology in children living in these circumstances. Tungiasis is a disease of the poor."

Plan International, an aid organization, is distributing detergents such as potassium permanganate solution in Ugandan villages as a disinfectant, while medical officials in the affected regions are urging villagers to improve their hygiene.
The Uganda government has allocated US\$1 million to fight the epidemic and, according to a press release, has asked four cabinet ministers to oversee "the extermination of the parasites" from the affected region (http://pressrelease .co.ug/pressuganda/? $\mathrm{p}=2111$ ).

Poverty and a lack of transportation will be among the obstacles.

"I received treatment only once when I could still afford to walk to a referral hospital, which is 20 kilometres from my village, but things have changed since my legs started swelling and rotting," Samuel Munagwa told reporters on a field trip to the affected regions.

Another sufferer, Israel Kagwa, said on the same field trip that he has never received any treatment and that he's too weak to walk to the hospital. Kagwa, who's also suffering from elephantiasis, added that "my feet are rotting away. The pain is too much but what can I do about it? I need help."

It's not the first major outbreak of jiggers in Uganda. Previous outbreaks have even prompted unusual political responses, like one in 2008 in which a Member of Parliament called for the arrest of people infected with jiggers for ostensibly flaunting public health laws. - Kennedy Jawoko, Kampala, Uganda

CMAJ 2011.DOI:10.1503/cmaj.109-3726 